\title{
Pre-treatment neutrophil to lymphocyte ratio as a prognostic marker to predict chemotherapeutic response and survival outcomes in metastatic advanced gastric cancer
}

\author{
In Rae Cho $\cdot$ Jun Chul Park $\cdot$ Chan Hyuk Park $\cdot$ Jung Hyun Jo • \\ Hyun Jik Lee $\cdot$ Sunyong Kim $\cdot$ Choong Nam Shim $\cdot$ \\ Hyuk Lee $\cdot$ Sung Kwan Shin $\cdot$ Sang Kil Lee $\cdot$ Yong Chan Lee
}

Received: 21 July 2013/Accepted: 16 December 2013/Published online: 19 January 2014

(C) The International Gastric Cancer Association and The Japanese Gastric Cancer Association 2014

\begin{abstract}
Background Several studies have shown that the neutrophil to lymphocyte ratio (NLR) in peripheral blood is a prognostic factor of various cancers. However, there is limited information on the clinical and prognostic significance of NLR in patients with metastatic advanced gastric cancer (AGC). Therefore, we examined whether the NLR can be used as a prognostic marker for predicting chemotherapeutic response and survival outcomes in metastatic AGC patients who are receiving palliative chemotherapy. Method A total of 268 patients diagnosed with metastatic AGC were enrolled. NLR was calculated from complete blood cell count taken before the first chemotherapy treatment. Patients were divided into two groups according to the median value of NLR: a high NLR group and a low NLR group.

Result The median follow-up period was 340 days (range 72-1796 days) and median NLR was 3.06 (range 0.18-18.16). The high NLR group (NLR $>3.0$ ) contained 138 patients and the low NLR group (NLR $\leq 3.0)$ contained 130 patients. Low NLR group patients had a significantly higher chemotherapeutic disease control rate $(90.0 \%$ vs. 80.4; $P=0.028$ ), and longer progression-free survival (PFS) and overall survival (OS) than the high NLR group patients (186 vs. 146 days; $P=0.001 ; 414$ vs. 280 days; $P<0.001$, respectively). In multivariate analysis, NLR showed a significant association with PFS (HR 1.478; $95 \%$
\end{abstract}

I. R. Cho · J. C. Park $(\varangle) \cdot$ C. H. Park ·

J. H. Jo - H. J. Lee - S. Kim - C. N. Shim - H. Lee

S. K. Shin - S. K. Lee - Y. C. Lee

Department of Internal Medicine and Institute of Gastroenterology, Severance Hospital, Yonsei University

College of Medicine, 250 Seongsanno, Seodaemun-gu,

Seoul 120-752, Republic of Korea

e-mail: junchul75@yuhs.ac
CI $1.154-1.892 ; P=0.002)$ and OS (HR $1.569 ; 95 \% \mathrm{CI}$ 1.227-2.006; $P<0.001)$.

Conclusion Pretreatment NLR is a useful prognostic marker in patients with metastatic AGC who are undergoing palliative chemotherapy.

Keywords Gastric cancer · Chemotherapy · Survival

\section{Introduction}

Advanced gastric cancer (AGC) is one of the most common causes of malignancy and cancer-related deaths worldwide, with a particularly high incidence in Asian countries. In particular, metastatic AGC patients have the worst prognosis among gastric cancer patients and a very low 5-year survival rate [1]. Although systemic chemotherapy is the mainstay of treatments for patients with metastatic AGC, survival outcome for palliative chemotherapy varies among patients. In addition, in clinical practice adverse effects of chemotherapy also potentially reduce the quality of life in these patients. Therefore, it is essential to identify patients who are more likely to benefit from palliative chemotherapy, which could then guide treatment strategies and potentially reduce the side effects and costs associated with an ineffective therapy and eventually improve survival outcomes. However, a reliable prognostic marker that can accurately predict the survival outcome of metastatic gastric cancer patients, who are receiving palliative chemotherapy, has not been identified.

Recently, there has been growing interest in the host's inflammatory response to tumors and the systemic effects of tumors that cause up-regulation of the inflammatory process. This tumor-generated inflammatory response may result in an increased propensity for metastasis via up- 
regulation of cytokine and inflammatory mediators, inhibition of apoptosis, promotion of angiogenesis, and damage to DNA [2]. The systemic inflammatory response also represents changes in the relative levels of circulating white blood cells, with an increase in neutrophils and decrease in lymphocytes with the advancement of cancer [3].

In recent studies, the neutrophil to lymphocyte ratio (NLR) in the peripheral blood has been shown to be a prognostic factor in various kinds of cancers including colorectal cancer, non-small cell lung cancer, and hepatocellular carcinoma [4-7]. However, only limited data on the clinical and prognostic significance of NLR in patients with gastric cancer have been reported [8-11], and most of the previous studies focused on NLR as a prognostic factor in operable early-stage AGC patients with limited data in metastatic AGC patients $[9,11]$. Furthermore, there are no reports on the relationship between NLR and chemotherapeutic response in metastatic gastric cancer.

Therefore, we aimed to evaluate whether the NLR can be used as a prognostic marker for predicting chemotherapeutic response and survival outcome in metastatic AGC patients receiving palliative chemotherapy.

\section{Materials and methods}

Study population

Medical records from patients diagnosed with metastatic AGC in the Severance Hospital, Yonsei University College of Medicine between January 2006 and November 2009 were retrospectively reviewed. All patients were histologically confirmed and underwent palliative chemotherapy. The inclusion criteria were as follows: (1) age 18 to 80 years; (2) histologically-proven inoperable advanced gastric adenocarcinoma; (3) at least one measurable or evaluable lesion; (4) Eastern Cooperative Oncology Group (ECOG) performance status $\leq 2$; (5) no prior antitumor treatment, such as chemotherapy, surgery, or radiotherapy; (6) estimated life expectancy $\geq 12$ weeks; (7) adequate bone marrow function (absolute neutrophil count $\geq 1.5 \times 10^{9} / \mathrm{L}$ and platelet count $\geq 100 \times 10^{9} / \mathrm{L}$ ); (8) adequate renal function (serum creatinine $<1.5 \mathrm{mg} / \mathrm{dL}$ or calculated creatinine clearance $\geq 60 \mathrm{~mL} / \mathrm{min}$ by the Cockcroft and Gault formula); and (9) adequate hepatic function [total bilirubin level $\leq 2$ times institutional upper limit of normal (ULN) and serum transaminase level $\leq 3$ times the institutional ULN]. Finally, 268 patients were enrolled in this study.

All subjects underwent chest radiography, laboratory tests, esophago-gastroduodenoscopy (EGD) with biopsy, bone scintigraphy (if needed), and an abdominal pelvic computed tomography (CT) or ${ }^{18} \mathrm{~F}$-fluorodeoxyglocuse- positron emission tomography $\left({ }^{18} \mathrm{~F}-\mathrm{FDG}-\mathrm{PET}\right) / \mathrm{CT}$ scan. History taking and physical examination revealed no systemic infection and fever in patients at baseline. Gastric carcinomas were assessed histologically and classified using the World Health Organization (WHO) criteria for gastric cancer [12]. Well or moderately differentiated adenocarcinomas were classified as differentiated adenocarcinomas. Poorly differentiated adenocarcinomas or signet ring cell carcinomas (SRC) were classified as undifferentiated adenocarcinomas.

This study is approved by the independent institutional review board, and the study protocol is consistent with the ethical guidelines of the 1975 Declaration of Helsinki.

\section{Peripheral blood parameters}

Venous blood was sampled before the first chemotherapy treatment and collected in ethylenediaminetetraacetic acid (EDTA) containing tubes. Blood cell counts were analyzed using a standard Coulter1 counter (Model, XE2100, Sysmex Co., Kobe, Japan). The normal range of white blood cell count (WBC) was from 4,000 to 10,800 cells $/ \mathrm{mm}^{3}$. Baseline NLR was calculated as neutrophil count divided by lymphocyte count. The patients were dichotomized at the median value of NLR.

Treatments and response evaluation

All patients underwent palliative chemotherapy after diagnosis. The most commonly used chemotherapeutic regimen was a taxane (paclitaxel or docetaxel)-based doublet regimen $(n=115,43 \%)$, followed by FOLFOX (oxaliplatin, 5-flurouracil, and leucovorin), platinum based and a CPT-11 based regimen. The chemotherapy regimen was decided at the discretion of the physician and was not related to the degree of the disease.

Depending on the drug regimen, treatment responses were evaluated by ${ }^{18} \mathrm{~F}$-FDG-PET/CT or contrast-enhanced $\mathrm{CT}$. In patients treated with regimens performed every 2 weeks, response evaluation was done every four cycles. For patients treated with regimens on a 3-week or 4-week interval, the response evaluation was carried out every two cycles. About 1 week variation is regarded as allowable error. Gastroduodenoscopy, ultrasonography, and bone scintigraphy were performed when clinically indicated. Follow-up evaluation also included a complete physical examination, biochemical screening, and chest X-ray. We adopted the response evaluation criteria in solid tumors (RECIST) [13] as follows: complete response (CR), partial response (PR), stable disease (SD), and progressive disease (PD) [14]. Objective response was defined as CR or PR, while disease control was defined as CR, PR, or SD. Response was analyzed by intention-to-treat analysis. 
Progression-free survival (PFS) was defined as the interval between the date of chemotherapy initiation and the date of cancer progression. Overall survival (OS) was defined as the interval between the date of chemotherapy initiation and the date of death or last contact.

Statistical analysis

All statistical analyses were performed using SPSS for Windows version 18.0 (SPSS, Inc., Chicago, IL). The independent $t$ test and Chi Square test were used to analyze differences among groups of patients with high or low NLR. Survival curves were estimated by the Kaplan-Meier method, and the difference between the curves was assessed by the log rank test. Multivariate analysis was performed to determine the significance of prognostic variables using Cox proportional hazards method. A $P$ value of $<0.05$ was considered statistically significant.

\section{Results}

Baseline characteristics

The median follow-up period was 340 days (range 72-1796 days), and median NLR was 3.06 (range 0.18-18.16). Baseline characteristics of the 268 patients are shown in Table 1. The mean age was 55.4 years, and 175 $(65.3 \%)$ patients were male. A distant lymph node was the most frequent site for metastasis (187 patients, $69.8 \%$ ),

Table 1 Baseline characteristics of patients

\begin{tabular}{|c|c|c|c|c|}
\hline Variables & Total patients $(n=268)$ & Low NLR group $(n=130)$ & High NLR group $(n=138)$ & $P$ \\
\hline Age, years & $55.44( \pm 12.48)$ & $57.65( \pm 11.47)$ & $53.36( \pm 13.06)$ & 0.004 \\
\hline Sex & & & & 0.291 \\
\hline Male & $175(65.3 \%)$ & $89(68.5 \%)$ & $86(62.3 \%)$ & \\
\hline Female & $93(34.7 \%)$ & $41(31.5 \%)$ & $52(37.7 \%)$ & \\
\hline ECOG & & & & $0.944^{\mathrm{c}}$ \\
\hline 0 & $135(50.4 \%)$ & $67(51.5 \%)$ & $68(49.3 \%)$ & \\
\hline 1 & $126(47.0 \%)$ & $60(46.2 \%)$ & $66(47.8 \%)$ & \\
\hline 2 & $7(2.6 \%)$ & $3(2.3 \%)$ & $4(2.9 \%)$ & \\
\hline Pathology & & & & 0.371 \\
\hline Differentiated & $95(35.4 \%)$ & $50(38.5 \%)$ & $45(32.6 \%)$ & \\
\hline Undifferentiated & $173(64.6 \%)$ & $80(61.5 \%)$ & $93(67.4 \%)$ & \\
\hline \multicolumn{5}{|l|}{ Location of metastasis } \\
\hline 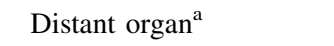 & $150(56.0 \%)$ & $68(52.3 \%)$ & $82(59.4 \%)$ & 0.241 \\
\hline Distant lymph node ${ }^{\mathrm{b}}$ & $187(69.8 \%)$ & $85(65.4 \%)$ & $102(73.9 \%)$ & 0.129 \\
\hline Peritoneum & $157(58.6 \%)$ & $69(53.1 \%)$ & $88(63.8 \%)$ & 0.077 \\
\hline \multicolumn{5}{|l|}{ Chemotherapeutic regimen } \\
\hline Taxane-based & $115(42.9 \%)$ & $57(43.8 \%)$ & $58(42.0 \%)$ & \\
\hline FOLFOX & $76(28.4 \%)$ & $41(31.5 \%)$ & $35(25.4 \%)$ & \\
\hline Platinum-based & $56(20.9 \%)$ & $25(19.2 \%)$ & $31(22.5 \%)$ & \\
\hline CPT-11-based & $21(7.8 \%)$ & $7(5.4 \%)$ & $14(10.1 \%)$ & \\
\hline CEA (ng/mL) & $68.11( \pm 341.98)$ & $33.10( \pm 109.48)$ & $101.08( \pm 463.01)$ & 0.096 \\
\hline CA $19-9(\mathrm{U} / \mathrm{mL})$ & $804.73( \pm 2875.93)$ & $542.28( \pm 2027.47)$ & $1055.52( \pm 3489.57)$ & 0.143 \\
\hline WBC (cells $/ \mathrm{mm}^{3}$ ) & $7524.70( \pm 2238.26)$ & $6699.46( \pm 1719.40)$ & $8302.10( \pm 2392.69)$ & $<0.001$ \\
\hline Neutrophils (cells $/ \mathrm{mm}^{3}$ ) & $5146.08( \pm 2051.71)$ & $3994.31( \pm 1306.91)$ & $6231.09( \pm 2037.41)$ & $<0.001$ \\
\hline Lymphocytes (cells/mm³) & $1655.45( \pm 591.58)$ & $1962.23( \pm 588.79)$ & $1366.45( \pm 426.83)$ & $<0.001$ \\
\hline Hemoglobin (g/dL) & $11.49( \pm 2.30)$ & $11.65( \pm 2.31)$ & $11.34( \pm 2.29)$ & 0.262 \\
\hline Platelets $\left(10^{3}\right.$ cells $\left./ \mathrm{mm}^{3}\right)$ & $349.74( \pm 116.27)$ & $334.95( \pm 92.42)$ & $363.66( \pm 133.80)$ & 0.041 \\
\hline
\end{tabular}

Variables are expressed as mean $( \pm \mathrm{SD})$ or $n(\%)$

$N L R$ neutrophil to lymphocyte ratio, ECOG Eastern Cooperative Oncology Group, $C E A$ carcinoembriogenic antigen, $C A$ carbohydrate antigen, $W B C$ white blood cell count

${ }^{a}$ Including liver, lung, ovary, pancreas, colon, and bone

b Paraaortic, superior mesenteric artery, retroperitoneal, and supraclavicular lymph node

c By Fisher's exact test 
followed by peritoneum $(157,58.6 \%)$ and distant organ (liver, lung, ovary, pancreas, colon, and bone) (150, $56.0 \%)$.

We determined the cut-off value of NLR for prognostic stratification. Based on the median value of NLR, the patients were divided into two groups (Fig. 1): high NLR group (NLR $>3$ ) and low NLR group (NLR $\leq 3$ ). Low NLR group patients were significantly older than high NLR group patients $(P=0.004)$. Low NLR group patients had a lower white blood cell count $(P<0.001)$ and neutrophil count $(P<0.001)$ than high NLR group patients. Lymphocyte count was higher in low NLR patients than in high NLR group patients $(P<0.001)$. Platelet count was higher in high NLR patients $(P=0.041)$. Other clinicopathological characteristics were not significantly different between the two groups.
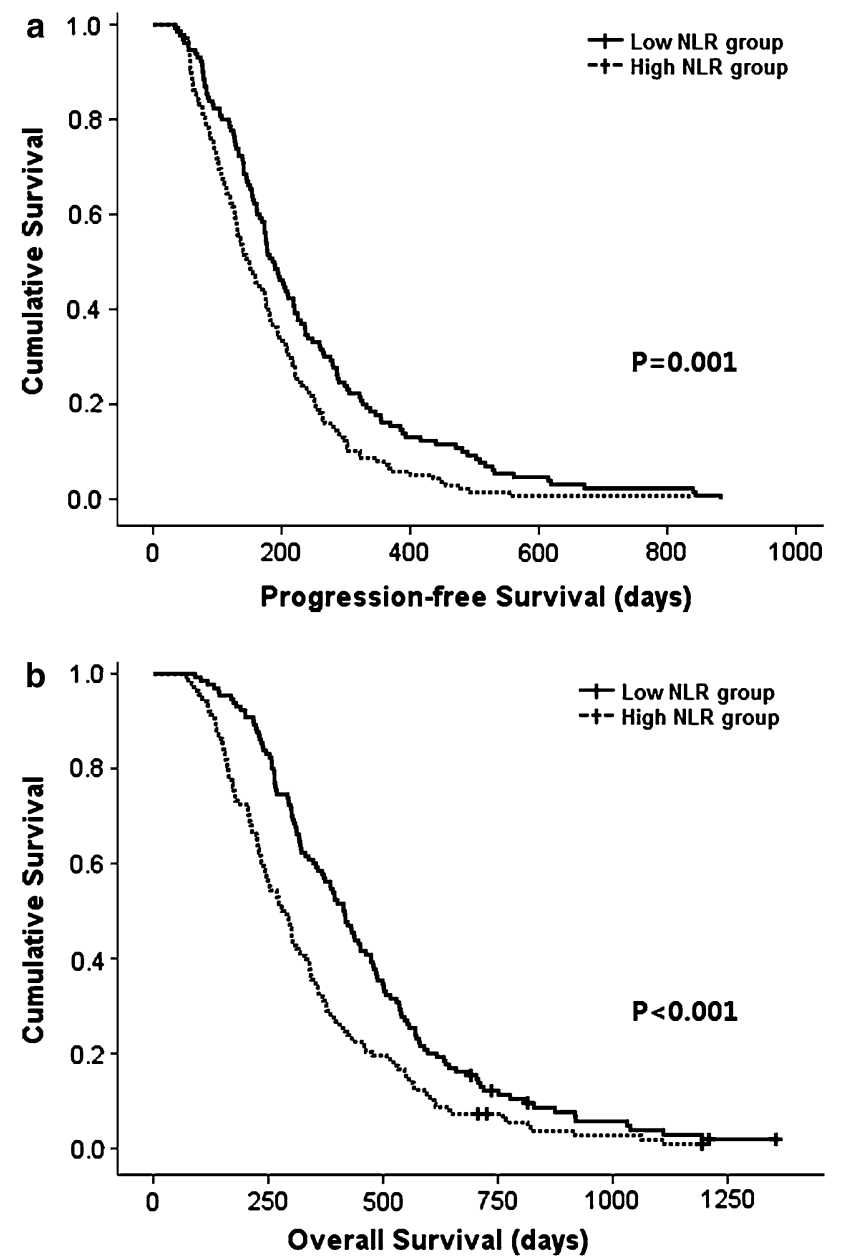

Fig. 1 Kaplan-Meier survival curves for a progression-free survival and $\mathbf{b}$ overall survival of patients (high and low NLR patients). Median PFS and OS were longer in the low NLR group than in the high NLR group [PFS 186 days (95\% CI 162.91-209.09) vs. 146 days $(122.15-169.85), \quad P=0.001 ; \quad$ OS 414 days (372.50-455.50) vs. 280 days $(243.16-316.84), P<0.001]$
Table 2 Treatment response to chemotherapy according to NLR

\begin{tabular}{|c|c|c|c|}
\hline Response & $\begin{array}{l}\text { Total patients } \\
(n=268)\end{array}$ & $\begin{array}{l}\text { Low NLR group } \\
(n=130)\end{array}$ & $\begin{array}{l}\text { High NLR group } \\
(n=138)\end{array}$ \\
\hline \multicolumn{4}{|c|}{ Non-progression of disease ${ }^{\dagger}$} \\
\hline Complete response & $0(0 \%)$ & $0(0 \%)$ & $0(0 \%)$ \\
\hline Partial response & $127(47.4 \%)$ & $62(47.7 \%)$ & $65(47.1 \%)$ \\
\hline Stable disease & $101(37.7 \%)$ & $55(42.3 \%)$ & $46(33.3 \%)$ \\
\hline Progressive disease & $40(14.9 \%)$ & $13(10.0 \%)$ & $27(19.6 \%)$ \\
\hline
\end{tabular}

${ }^{\dagger} P=0.028$ for disease control rate between the low NLR group and the high NLR group

$N L R$ neutrophil to lymphocyte ratio

Chemotherapeutic response

The distribution of the chemotherapeutic response after the chemotherapy with reference to NLR subgroup is shown in Table 2. Overall, 0 and 127 patients $(47.4 \%)$ had CR and PR, while $101(37.7 \%)$ and $40(14.9 \%)$ patients had SD and PD, respectively. The objective response rates were not different according to the NLR (47.7 vs. $47.1 \%$ $P=0.923)$. However, the low NLR group had a significantly higher disease control rate than the low NLR group (90 vs. $80.4 \%, P=0.028$ ).

Progression-free and overall survival

The median PFS and OS were173 days [95 \% confidence interval (CI), 158.56-187.43] and 338 days (95\% CI 304.19-371.81), respectively. Median PFS was longer in the low NLR group than in the high NLR group [186 days (95\% CI 162.91-209.09) vs. 146 days (95\% CI 122.15-169.85), $P=0.001$; Fig. 1a]. Moreover, the median OS was also longer in the low NLR group than in the high NLR group [414 days (95\% CI 372.50-455.50) vs. 280 days (95 \% CI 243.16-316.84), $P<0.001$; Fig. 1b].

Clinicopathological variables for prediction of prognosis were tested in univariate and multivariate analyses (Tables 3, 4). Univariate predictors of PFS were NLR $(P=0.001)$ and response to first-line chemotherapy $(P<0.001)$. In multivariate analysis, NLR [hazard ratio (HR) $1.478,95 \%$ CI 1.154-1.892; $P=0.002]$ and response to first-line chemotherapy (HR 51.708, $95 \%$ CI 27.273-98.034; $P<0.001)$ were also independent predictors of PFS.

Univariate predictors of OS were NLR $(P<0.001)$, tumor differentiation $(P=0.025)$, and response to firstline treatment $(P<0.001)$. In multivariate analysis, NLR (HR 1.569, $95 \%$ CI 1.227-2.006; $P<0.001$ ), tumor differentiation (HR 1.379, $95 \%$ CI 1.066-1.784; $P=0.014$ ) and response to first-line chemotherapy (HR 3.356, $95 \%$ CI 2.361-4.770; $P<0.001)$ were independent predictors of OS. 
Table 3 Results of univariate and multivariate analyses of PFS

\begin{tabular}{|c|c|c|c|}
\hline \multirow[t]{2}{*}{ Variables } & \multirow{2}{*}{$\begin{array}{l}\text { Univariate } \\
\text { analysis } \\
P \text { value }\end{array}$} & \multicolumn{2}{|l|}{ Multivariate analysis } \\
\hline & & $\mathrm{HR}(95 \% \mathrm{CI})$ & $P$ value \\
\hline \multicolumn{4}{|l|}{ Age } \\
\hline$\leq 60$ & 0.193 & & \\
\hline$>60$ & & & \\
\hline \multicolumn{4}{|l|}{ Sex } \\
\hline Male & 0.367 & & \\
\hline \multicolumn{4}{|l|}{ Female } \\
\hline \multicolumn{4}{|l|}{ ECOG } \\
\hline 0 & 0.512 & & \\
\hline 1 & & & \\
\hline 2 & & & \\
\hline Hemoglobin (g/dL) & 0.564 & & \\
\hline Platelet $\left(\right.$ cells $\left./ \mathrm{mm}^{3}\right)$ & 0.491 & & \\
\hline \multicolumn{4}{|l|}{ NLR } \\
\hline Low & 0.001 & 1.000 & 0.002 \\
\hline High & & $1.478(1.154-1.892)$ & \\
\hline \multicolumn{4}{|l|}{ Pathology } \\
\hline Differentiated & 0.106 & & \\
\hline Undifferentiated & & & \\
\hline \multicolumn{4}{|c|}{ Distant organ and peritoneal metastasis } \\
\hline Negative & 0.097 & & \\
\hline \multicolumn{4}{|l|}{ Positive } \\
\hline \multicolumn{4}{|l|}{ Distant LN metastasis } \\
\hline Negative & 0.340 & & \\
\hline \multicolumn{4}{|l|}{ Positive } \\
\hline \multicolumn{4}{|c|}{ Chemotherapeutic regimen } \\
\hline Taxane-based & 0.394 & & \\
\hline FOLFOX & & & \\
\hline Platinum-based & & & \\
\hline CPT-11-based & & & \\
\hline \multicolumn{4}{|c|}{ Response to first-line chemotherapy } \\
\hline $\mathrm{PR} / \mathrm{SD}$ & $<0.001$ & 1.000 & $<0.001$ \\
\hline PD & & $51.708(27.273-98.034)$ & \\
\hline
\end{tabular}

ECOG Eastern Cooperative Oncology Group, $L N$ lymph node, $P R$ partial response, $S D$ stable disease, $P D$ progressive disease, $N L R$ neutrophil to lymphocyte ratio

\section{Discussion}

This study shows that pre-treatment NLR in the peripheral blood of patients with metastatic AGC can be used as a prognostic marker to predict chemotherapeutic response and survival time. Although several studies have shown a relationship between NLR and stomach cancer prognosis $[10,11,15,16]$, previous studies have mainly focused on postoperative survival in operable AGC patients rather than metastatic AGC patients. Furthermore, there are no reports on the relationship between NLR and therapeutic response in gastric cancer, even though prediction of
Table 4 Results of univariate and multivariate analyses of OS

\begin{tabular}{|c|c|c|c|}
\hline \multirow[t]{2}{*}{ Variables } & \multirow{2}{*}{$\begin{array}{l}\text { Univariate } \\
\text { analysis } \\
P \text { value }\end{array}$} & \multicolumn{2}{|l|}{ Multivariate analysis } \\
\hline & & $\mathrm{HR}(95 \% \mathrm{CI})$ & $P$ value \\
\hline \multicolumn{4}{|l|}{ Age } \\
\hline$\leq 60$ & 0.574 & & \\
\hline$>60$ & & & \\
\hline \multicolumn{4}{|l|}{ Sex } \\
\hline Male & 0.200 & & \\
\hline Female & & & \\
\hline \multicolumn{4}{|l|}{ ECOG } \\
\hline 0 & 0.589 & & \\
\hline 1 & & & \\
\hline 2 & & & \\
\hline Hemoglobin (g/dL) & 0.839 & & \\
\hline Platelet (cells $/ \mathrm{mm}^{3}$ ) & 0.335 & & \\
\hline \multicolumn{4}{|l|}{ NLR } \\
\hline Low & $<0.001$ & 1.000 & $<0.001$ \\
\hline High & & $1.569(1.227-2.006)$ & \\
\hline \multicolumn{4}{|l|}{ Pathology } \\
\hline Differentiated & 0.025 & 1.000 & 0.014 \\
\hline Undifferentiated & & $1.379(1.066-1.784)$ & \\
\hline \multicolumn{4}{|c|}{ Distant organ and peritoneal metastasis } \\
\hline Negative & 0.192 & & \\
\hline Positive & & & \\
\hline \multicolumn{4}{|l|}{ Distant LN metastasis } \\
\hline Negative & 0.570 & & \\
\hline Positive & & & \\
\hline \multicolumn{4}{|c|}{ Chemotherapeutic regimen } \\
\hline Taxane-based & 0.309 & & \\
\hline FOLFOX & & & \\
\hline Platinum-based & & & \\
\hline CPT-11-based & & & \\
\hline \multicolumn{4}{|c|}{ Response to first-line chemotherapy } \\
\hline $\mathrm{PR} / \mathrm{SD}$ & $<0.001$ & 1.000 & $<0.001$ \\
\hline PD & & $3.356(2.361-4.770)$ & \\
\hline
\end{tabular}

ECOG Eastern Cooperative Oncology Group, $L N$ lymph node, $P R$ partial response, $S D$ stable disease, $P D$ progressive disease, $N L R$ neutrophil to lymphocyte ratio

chemotherapeutic response in metastatic AGC patients is clinically important. To the best of our knowledge, this is the first study assessing the value of the NLR as a prognostic marker for prediction of chemotherapeutic response in metastatic AGC patients who are receiving palliative chemotherapy. Our findings showed that NLR is a useful prognostic marker in these patients.

In recent decades, our understanding of the inflammatory microenvironment of cancer has increased rapidly, and there is growing interest in the relationship between inflammation and cancer. Consequently, various indicators that reflect the relationship between cancer and 
inflammation have been researched and developed. Recently reported predictive inflammatory markers include interferon-gamma/interleukin-4 ratio [15], endothelial growth factor per platelet count [17], and inflammationbased prognostic score (Glasgow prognostic score, GPS) based on albumin and CRP levels [18].

NLR also reflects systemic inflammation and previous results support the use of NLR as predictive inflammatory markers and as an independent predictor of clinical benefit in patients receiving chemotherapy [10]. Our study also demonstrates that a longer PFS $(P=0.001$; Fig. 1a) and OS $(P<0.001$; Fig. 1b) in the low NLR group than in the high NLR group. In addition, NLR showed a significant relationship with both PFS and OS in multivariate analysis. Similar to our findings, previous studies also showed that high baseline NLR predicts poor survival in patients with operable gastric cancer $[10,11,16]$. These studies indicated that NLR could be a simple and reliable prognostic factor for risk stratification and might provide better treatment allocation in gastric cancer. There are several possible explanations for the association between elevated NLR and poor cancer prognosis. One explanation is decreased lymphocyte count and function. Lymphocytes are a crucial component of the host's cellular adaptive immunity against cancer cells and can attack cancer cells and eliminate nascent tumor cells [19]. Patients with lymphocyte infiltration around the tumor may have a better prognosis than those without infiltration [20, 21], and a clinical study has demonstrated that a low total lymphocyte count can be used as an index of an adverse outcome in pancreatic cancer [22]. In our study, we also evaluated the relationship for absolute lymphocyte count and survival. The high absolute lymphocyte count group showed longer PFS than those of lower than median group (PFS 181 vs. 154 days; $P<0.001$ ). There is also a high tendency that the low absolute lymphocyte count group showed longer OS than the high absolute lymphocyte count group (OS 371 vs. 294 days; $P=0.050$ ).

Another possible explanation is increased neutrophil number and function. Several lines of evidence suggest that neutrophils may actually promote cancer cell growth and metastasis and/or suppress lymphocyte activity [23]. Circulating neutrophils have been shown to contain and secrete pro-angiogenic factors including vascular endothelial growth factor (VEGF). Therefore, an elevated neutrophil count stimulates tumor angiogenesis and aids the progression of neoplasm $[24,25]$. In our study, there was no significant difference shown in PFS according to absolute neutrophil count (PFS 179 vs. 161 days; $P=0.250$ ). However, a tendency of longer OS was shown in the low absolute neutrophil count group compared with the high absolute neutrophil count group (OS 376 vs. 303 days; $P=0.077$ ).
Interestingly, in our study, the low NLR group had a significantly higher disease control rate than the high NLR group (90 vs. $80.4 \%, P=0.028$ ). Although prediction of chemotherapeutic response in metastatic cancer patients is important, there are insufficient data on novel markers predicting chemotherapeutic response. To date, several studies have investigated efficient predictive markers for treatment response. One study reported that tumor differentiation and tumor size could be used as a clinical predicator of pathologic tumor response to neoadjuvant chemotherapy in patients with locally advanced gastric cancer [26]. Gene methylation status was also reported as a predicator of chemotherapy response in gastric cancer [27, 28]. However, these assessments are limited in terms of their cost-effectiveness and requirement for an additional blood test, genetic specialists, or expensive equipment. In contrast, NLR is a simple, readily available method and cost-effective compared with pathologic evaluation or genetic analysis. However, there are few clinical studies showing the relationship between NLR and chemotherapeutic response in metastatic cancers. One cohort study with 349 colorectal cancer patients showed a relationship between NLR and chemotherapeutic response. In that study, low NLR was associated with improved clinical benefit and the NLR was confirmed to independently predict OS in the validation cohort [29].

Immunological response and signaling of NLR in gastric cancer chemotherapy might be explained by several hypotheses. In the first place, immunologic surveillance against tumor cells is reported to be an important microenvironment to prevent tumor progression. Consistent with the fact that the acquired immunity is eventually mediated by lymphocytes, several investigators had already reported the better prognosis in solid cancers when the infiltration of lymphocytes around the tumor tissues is observed [20,21]. In a similar context, the lower NLR (relative "lymphocyte dominant") at baseline might be translated into the more "favorable" condition for subsequent anti-tumor immunologic reaction. Furthermore, when cytotoxic chemotherapy is administered, tumor necrosis occurs, leading to subsequent inflammatory reaction including stimulation of antigen presentation by tumor-infiltrating dendritic cells and subsequent induction of an antitumor immune response. At this time, patients with the lower NLR are likely to have the milieu of "better" inflammatory reaction mediated by lymphocytes compared to those with the higher NLR.

The cut-off value of NLR used in the present study was based upon the median value of all patients. A previous study with 293 AGC patients who had undergone gastrectomy reported that the median NLR value was 2.06, and high NLR patients showed worse overall survival than low NLR patients based on this median cut-off value. 
When the cut-off value was moved to 3.0, the value of the 75th percentile, there was a significant relationship between NLR and disease-free survival [10]. In another study with 1028 AGC patients who had a median NLR value of 2.33, a NLR of 4.0 was suggested to be the ideal cut-off value, but NLR 3.0 was also statistically significant for predicting the patients overall survival [11]. Although more evidence is needed to confirm the ideal cut-off value, a NLR of 3.0 can be considered a reliable predictive value for prognosis in gastric cancer patients based on these studies.

Clinically, NLR can help stratification of patients with metastatic AGC who are receiving palliative chemotherapy. Prognostic stratification based on pre-treatment NLR may guide physicians to incorporate a tailored treatment regimen based on the patients' performance status. For example, physicians may intensify the treatment regimens or add another drug such as a molecular targeted agent in the poor prognosis group with good performance status. Further studies are needed to validate this presumption.

This study has some limitations. First, the study has a relatively small sample size and retrospective design. Study populations in the present study do not represent overall patients with metastatic AGC. In particular, there was a lack of data on drug administration history that might influence lymphocyte count (e.g., NSAIDS, aspirin, corticosteroids, and other anti-inflammatory drugs). Therefore, the results of our study should be confirmed through a large-scale, prospective study. Second, identical chemotherapeutic regimens were not administered to all patients in this study, although more than $40 \%$ of the patients underwent combination chemotherapy with a taxane-based doublet regimen and the other chemotherapeutic regimens had been reported to demonstrate comparable clinical efficacy. Because of the heterogeneity of chemotherapeutic regimens, the timing of the response evaluation was not constant. Finally, we could not conduct histological examinations to investigate the relationship between tissue neutrophila (and/or lymphopenia) and systemic neutrophilia (and/or lymphopenia). The reaction of host inflammatory cells against cancer cells consists of both locoregional and systemic responses. Therefore, further investigation is needed to clarify the relationship between tissue neutrophilia and systemic neutrophilia.

In conclusion, pre-treatment NLR can be used as a prognostic marker to predict chemotherapeutic response, PFS, and OS in patients with metastatic AGC who are undergoing palliative chemotherapy. Measurement of NLR before treatment is an inexpensive, reproducible, and widely available blood test that may provide a simple method of identifying patients with a poorer prognosis and help to effectively guide treatment.
Conflict of interest None of the authors have any conflicts of interest related to this study.

\section{References}

1. Howlader N, Noone AM, Krapcho M, Neyman N, Aminou R, Waldron W, et al, editors. National Cancer Institute. Bethesda, MD. Posted to the SEER web site. http://seer.cancer.gov. April 2012.

2. Balkwill F, Mantovani A. Inflammation and cancer: back to Virchow? Lancet. 2001;357:539-45.

3. Satomi A, Murakami S, Ishida K, Mastuki M, Hashimoto T, Sonoda M. Significance of increased neutrophils in patients with advanced colorectal cancer. Acta Oncol. 1995;34:69-73.

4. Gomez D, Farid S, Malik HZ, Young AL, Toogood GJ, Lodge JP, et al. Preoperative neutrophil-to-lymphocyte ratio as a prognostic predictor after curative resection for hepatocellular carcinoma. World J Surg. 2008;32:1757-62.

5. Halazun KJ, Aldoori A, Malik HZ, Al-Mukhtar A, Prasad KR, Toogood GJ, et al. Elevated preoperative neutrophil to lymphocyte ratio predicts survival following hepatic resection for colorectal liver metastases. Eur J Surg Oncol. 2008;34:55-60.

6. Kishi Y, Kopetz S, Chun YS, Palavecino M, Abdalla EK, Vauthey JN. Blood neutrophil-to-lymphocyte ratio predicts survival in patients with colorectal liver metastases treated with systemic chemotherapy. Ann Surg Oncol. 2009;16:614-22.

7. Sarraf KM, Belcher E, Raevsky E, Nicholson AG, Goldstraw P, Lim E. Neutrophil/lymphocyte ratio and its association with survival after complete resection in non-small cell lung cancer. J Thorac Cardiovasc Surg. 2009;137:425-8.

8. Bruckner HW, Lavin PT, Plaxe SC, Storch JA, Livstone EM. Absolute granulocyte, lymphocyte, and moncyte counts. Useful determinants of prognosis for patients with metastatic cancer of the stomach. JAMA. 1982;247:1004-6.

9. Yamanaka T, Matsumoto S, Teramukai S, Ishiwata R, Nagai Y, Fukushima M. The baseline ratio of neutrophils to lymphocytes is associated with patient prognosis in advanced gastric cancer. Oncology. 2007;73:215-20.

10. Jung MR, Park YK, Jeong O, Seon JW, Ryu SY, Kim DY, et al. Elevated preoperative neutrophil to lymphocyte ratio predicts poor survival following resection in late stage gastric cancer. J Surg Oncol. 2011;104:504-10.

11. Shimada H, Takiguchi N, Kainuma O, Soda H, Ikeda A, Cho A, et al. High preoperative neutrophil-lymphocyte ratio predicts poor survival in patients with gastric cancer. Gastric Cancer. 2010;13:170-6.

12. Hamilton SR, Aaltonen LA, Lambert R. Pathology and genetics of tumours of the digestive system. In: Kleihues P, Sobin L, editors. World Health Organization Classification of Tumours. Lyon: International Agency for Research on Cancer (IARC); 2000.

13. Eisenhauer EA, Therasse P, Bogaerts J, Schwartz LH, Sargent D, Ford R, et al. New response evaluation criteria in solid tumours: revised RECIST guideline (version 1.1). Eur J Cancer. 2009;45:228-47.

14. Therasse P, Arbuck SG, Eisenhauer EA, Wanders J, Kaplan RS, Rubinstein $\mathrm{L}$, et al. New guidelines to evaluate the response to treatment in solid tumors. European Organization for Research and Treatment of Cancer, National Cancer Institute of the United States, National Cancer Institute of Canada. J Natl Cancer Inst. 2000;92:205-16.

15. Ubukata H, Motohashi G, Tabuchi T, Nagata H, Konishi S. Evaluations of interferon-gamma/interleukin-4 ratio and 
neutrophil/lymphocyte ratio as prognostic indicators in gastric cancer patients. J Surg Oncol. 2010;102:742-7.

16. Dutta S, Crumley AB, Fullarton GM, Horgan PG, McMillan DC. Comparison of the prognostic value of tumour and patient related factors in patients undergoing potentially curative resection of gastric cancer. Am J Surg. 2012;204:294-9.

17. Seo HY, Park JM, Park KH, Kim SJ, Oh SC, Kim BS, et al. Prognostic significance of serum vascular endothelial growth factor per platelet count in unresectable advanced gastric cancer patients. Jpn J Clin Oncol. 2010;40:1147-53.

18. Crumley AB, McMillan DC, McKernan M, McDonald AC, Stuart RC. Evaluation of an inflammation-based prognostic score in patients with inoperable gastro-oesophageal cancer. Br J Cancer. 2006;94:637-41.

19. Smyth MJ, Dunn GP, Schreiber RD. Cancer immunosurveillance and immunoediting: the roles of immunity in suppressing tumor development and shaping tumor immunogenicity. Adv Immunol. 2006;90:1-50.

20. Kawata A, Une Y, Hosokawa M, Uchino J, Kobayashi H. Tumorinfiltrating lymphocytes and prognosis of hepatocellular carcinoma. Jpn J Clin Oncol. 1992;22:256-63.

21. Martinet L, Garrido I, Filleron T, Le Guellec S, Bellard E, Fournie JJ, et al. Human solid tumors contain high endothelial venules: association with T- and B-lymphocyte infiltration and favorable prognosis in breast cancer. Cancer Res. 2011;71:5678-87.

22. Fogar P, Sperti C, Basso D, Sanzari MC, Greco E, Davoli C, et al. Decreased total lymphocyte counts in pancreatic cancer: an index of adverse outcome. Pancreas. 2006;32:22-8.
23. Grivennikov SI, Greten FR, Karin M. Immunity, inflammation, and cancer. Cell. 2010;140:883-99.

24. Di Carlo E, Forni G, Musiani P. Neutrophils in the antitumoral immune response. Chem Immunol Allergy. 2003;83:182-203.

25. Shamamian P, Schwartz JD, Pocock BJ, Monea S, Whiting D, Marcus SG, et al. Activation of progelatinase A (MMP-2) by neutrophil elastase, cathepsin G, and proteinase-3: a role for inflammatory cells in tumor invasion and angiogenesis. J Cell Physiol. 2001;189:197-206.

26. Wang LB, Teng RY, Jiang ZN, Hu WX, Dong MJ, Yuan XM, et al. Clinicopathologic variables predicting tumor response to neoadjuvant chemotherapy in patients with locally advanced gastric cancer. J Surg Oncol. 2012;105:293-6.

27. Sugita H, Iida S, Inokuchi M, Kato K, Ishiguro M, Ishikawa T, et al. Methylation of BNIP3 and DAPK indicates lower response to chemotherapy and poor prognosis in gastric cancer. Oncol Rep. 2011;25:513-8.

28. Kato K, Iida S, Uetake H, Takagi Y, Yamashita T, Inokuchi M, et al. Methylated TMS1 and DAPK genes predict prognosis and response to chemotherapy in gastric cancer. Int $\mathrm{J}$ Cancer. 2008;122:603-8.

29. Chua W, Charles KA, Baracos VE, Clarke SJ. Neutrophil/lymphocyte ratio predicts chemotherapy outcomes in patients with advanced colorectal cancer. Br J Cancer. 2011;104:1288-95. 